\title{
REVIEW
}

\section{Triage of high-risk surgical patients for intensive care}

\author{
Julia B Sobol and Hannah Wunsch* \\ This article is one of eleven reviews selected from the Annual Update in Intensive Care and Emergency Medicine 2011 (Springer Verlag) and \\ co-published as a series in Critical Care. Other articles in the series can be found online at http://ccforum.com/series/annual. Further \\ information about the Annual Update in Intensive Care and Emergency Medicine is available from http://www.springer.com/series/8901
}

\begin{abstract}
Introduction
Patients who undergo high-risk non-cardiac surgical procedures represent a large proportion of admissions to intensive care units (ICUs) in the developed world [1]. Ideally, surgeons, anesthesiologists, and intensivists admitting surgical patients to ICUs target the patients who will benefit most from this highest level of postoperative care. However, accurately identifying which patients are at high risk of complications or death after major surgery remains difficult. For example, a recent study in the United Kingdom demonstrated that patients undergoing high-risk general surgical procedures comprised only $12.5 \%$ of surgical admissions to hospitals but over $80 \%$ of deaths, with less than $15 \%$ of these high-risk patients admitted to the ICU postoperatively [2].

Postoperative outcomes are a result of the complex interplay between the exact general surgical procedure performed, the previous health of the patient, and specific intra- and postoperative events. Outcomes may also be influenced by aspects of the particular healthcare system, such as the surgical procedure volume at different hospitals [3], as well as care options, such as the availability and suitable use of intensive care beds. Appropriate triage of patients to intensive care postoperatively may have a large impact on outcomes after non-cardiac surgery. This chapter reviews the patient factors and scoring systems developed to help with triage, describes current ICU triage recommendations for postsurgical patients, and identifies potential ways to improve evaluation and management of high-risk postoperative patients.
\end{abstract}

\section{Prediction of postoperative outcomes}

Predictors of postoperative outcomes may be divided into three categories: Known preoperative risk factors;

*Correspondence: hw2125@columbia.edu

Department of Anesthesiology, Columbia University, 622 West 168th Street, PH5-505, New York, NY 10032, USA the risk associated with the specific surgical procedure; and the unique aspects of each operative case that may contribute to a particular patient being at high risk for complications or death after surgery.

\section{Preoperative evaluation}

Many preoperative risk factors can help distinguish which patients are most likely to experience poor postoperative outcomes. In particular, preoperative comorbidities are well-established as predictors of both morbidity and mortality after surgery and can be measured in different ways (Table 1). Perhaps the best known is the American Society of Anesthesiologists' (ASA) physical status classification system. This is a widely used preoperative scoring system that describes the overall health of the patient and burden of comorbidities. The score is ideal in being simple to apply and requiring no laboratory data; however, it is also subject to substantial interobserver variation in score assignment [4]. Despite this inherent subjectivity, the ASA classification has been recognized as a helpful predictor of potential postoperative morbidity and mortality [5]. Another scoring system of preoperative comorbidities is the Charlson Comorbidity Index, which assigns weights to a variety of systemic diseases and predicts long-term survival [6]. While more detailed than the ASA classification, this score is usually considered more useful for research purposes than for risk stratification in real time. There are also organ system-specific scores, most notably the Revised Cardiac Risk Index (RCRI), a scoring system that incorporates six factors to predict the risk of major cardiac events after non-cardiac surgery [7]. None of these scoring systems alone is generally sufficient to provide adequate information regarding the risk for an individual patient. The RCRI predicts only cardiac risk, while the ASA and Charlson Comorbidity Index do not incorporate variables specific to the surgical procedure. 
Table 1. Select scoring systems available for assessment of postoperative risk

\begin{tabular}{|c|c|c|c|c|c|c|}
\hline Scoring system & Year & $\begin{array}{l}\text { Number of } \\
\text { variables }\end{array}$ & $\begin{array}{c}\text { Inclusion of } \\
\text { intraoperative } \\
\text { variables }\end{array}$ & Outcomes predicted & Simplicity & Objectivity \\
\hline \multicolumn{7}{|l|}{ Preoperative } \\
\hline ASA & 1941 & Unlimited & No & None & Simple & Subjective \\
\hline Charlson Comorbidity Index & 1987 & 19 & No & Mortality & Mildly complex & Objective \\
\hline RCRI & 1999 & 6 & No & Major cardiac complication & Simple & Objective \\
\hline \multicolumn{7}{|l|}{ Postoperative } \\
\hline P-POSSUM & 1998 & $\begin{array}{l}12 \text { physiologic, } \\
6 \text { operative }\end{array}$ & Yes & Morbidity \& mortality & Complex & Objective \\
\hline E-PASS & 2001 & $\begin{array}{l}6 \text { preoperative, } \\
3 \text { operative }\end{array}$ & Yes & Morbidity \& mortality & Complex & $\begin{array}{c}\text { Subjective } \\
\text { (includes ASA) }\end{array}$ \\
\hline NSQIP & 1997 & $\begin{array}{l}57 \text { preoperative, } \\
15 \text { operative }\end{array}$ & Yes & Morbidity \& mortality & Complex & $\begin{array}{c}\text { Subjective } \\
\text { (includes ASA) }\end{array}$ \\
\hline SAS & 2007 & 3 operative & Yes & Morbidity \& mortality & Simple & Objective \\
\hline \multicolumn{7}{|l|}{ Intensive care } \\
\hline APACHE I-IV* & 1981-2006 & $>10^{*}$ & No & Mortality & Complex & Objective \\
\hline SAPS I-III* & 1983-2005 & $>10^{*}$ & No & Mortality & Complex & Objective \\
\hline MPM I-III* & 1985-2007 & $>10^{*}$ & No & Mortality & Complex & Objective \\
\hline SOFA & 1994 & 6 & No & None & Mildly complex & Objective \\
\hline MODS & 1995 & 6 & No & None & Mildly complex & Objective \\
\hline
\end{tabular}

* Dependent on the version of the scoring system used

ASA: American Society of Anesthesiologists' physical status; RCRI: Revised Cardiac Risk Index; APACHE: Acute Physiology and Chronic Health Evaluation; SAPS: Simplified Acute Physiology Score; MPM: Mortality Probability Model; SOFA: Sequential Organ Failure Assessment; MODS: Multiple Organ Dysfunction Score; P-POSSUM: Portsmouth Physiological and Operative Severity Score for the enUmeration of Mortality and morbidity; E-PASS: Estimation of Physiologic Ability and Surgical Stress; NSQIP: National Surgical Quality Improvement Program; SAS: Surgical Apgar Score.

These preoperative scoring systems encompass a wide range of patient variables to produce a composite score. Individual patient factors have also been identified in large studies as independently predicting increased risk of morbidity and mortality after surgery, including age and poor nutritional and functional status $[8,9]$. Functional capacity at baseline may indicate how a patient will respond to surgical stress in the perioperative period. Recent guidelines from the American College of Cardiology Foundation (ACCF) and the American Heart Association (AHA) for preoperative cardiovascular risk evaluation in non-cardiac surgery rely on a modified version of the Duke Activity Status Index. This questionnaire estimates energy requirements for various patient activities. Depending on a patient's functional capacity, clinical risk factors, and type of surgery, the ACCF/AHA guidelines recommend either proceeding with surgery or further cardiac evaluation prior to the planned procedure. Clinical risk factors include active cardiac conditions and elements of the RCRI, and surgical procedures are divided into those that are low, intermediate, or high risk [10]. While these guidelines only address cardiovascular risk, they illustrate the importance of including both the patient's preoperative status and the particular surgical procedure for evaluation of the risk of poor postoperative outcomes.

\section{Intraoperative events}

Surgical duration and urgency have both been shown to impact postoperative outcomes, with longer duration and emergent procedures associated with worse outcomes $[2,9,11]$. Preoperative patient characteristics were the most important predictors of postoperative mortality in a large study of the Veterans Affairs database, but operative complexity became a significant predictor with highly complex procedures [12]. Pearse et al. defined high-risk surgical procedures as those with at least a $5 \%$ risk of mortality and found that these procedures, in combination with advanced age, comorbidities, and emergency surgery, were highly predictive of increased risk of death postoperatively [2]. While the weight of the effects of patient- and surgery-specific factors on postoperative outcomes varies across different studies, it is clear that it is ultimately the combination of the two that contributes to morbidity and mortality after surgery.

Surgical patients are unique as hospital patients in having a prolonged period of time in the operating room when they are closely monitored, providing detailed information on the physiologic perturbations associated with the anesthesia and surgery itself. The individual experience of a patient during surgery may have a large impact on outcomes. In particular, hemodynamic stresses to the body manifested as extremes of vital signs may 
influence the postoperative course. One study demonstrated that intraoperative tachycardia in long, complicated surgical procedures was independently associated with a composite measure of poor outcome [13]. In another case-control study, intraoperative tachycardia requiring treatment was associated with a significantly increased risk of ICU admission, prolonged hospital stay, and hospital mortality [14]. A prospective cohort study in elderly patients showed that ASA class, emergency surgery, and intraoperative tachycardia were the most important predictors of adverse outcomes after surgery [11]. These three studies indicate that intraoperative tachycardia is associated with a wide spectrum of poor postoperative outcomes. Unanswered questions raised by these studies are whether tachycardia represents inadequate preoperative beta-blockade for high-risk patients or whether better control of intraoperative tachycardia would improve postoperative outcomes.

In addition to heart rate, blood pressure extremes may also predict outcomes after surgery. A prospective evaluation of over 1,000 patients revealed only three significant independent predictors of one-year mortality after major general surgery: The Charlson Comorbidity Index, the cumulative deep hypnotic time, and intraoperative hypotension [15]. Other researchers found that the risk of one-year mortality in elderly patients may be influenced not only by the presence or absence of hypotension but by its duration as well. The cut-off value associated with an increased risk of one-year mortality was only 5 minutes for mean blood pressure less than $50 \mathrm{mmHg}$ but 30 minutes for mean blood pressure less than $60 \mathrm{mmHg}$ [16]. It is important to note, however, that intraoperative hypertension has also been associated with poor outcome [13], suggesting that it is not just a single hemodynamic response but many different types of physiologic stress that may affect postoperative morbidity and mortality in surgical patients.

\section{Perioperative scoring systems}

A number of scores aid in prediction of death specifically for patients admitted to the ICU (Table 1). While not developed solely for surgical patients, all of these scores account for postsurgical patients and provide risk prediction. The most commonly used scores are the Acute Physiology and Chronic Health Evaluation (APACHE) score, the Simplified Acute Physiology Score (SAPS), and the Mortality Probability Model (MPM) [17,18]. The Sequential Organ Failure Assessment (SOFA) and the Multiple Organ Dysfunction Score (MODS) are two other ICU scoring systems used to describe organ dysfunction over the course of the ICU stay $[19,20]$. The main problem with all of these scoring systems for triage of patients is that they were developed and validated on patients already admitted to the ICU, and they do not necessarily provide adequate prediction for a broader range of patients. Moreover, none of the ICU scoring systems takes into account specific intraoperative data from the surgical procedure itself.

Other scoring systems were created specifically for surgical populations to aid in postoperative predictions of morbidity and mortality, regardless of admission to the ICU. These scores incorporate patient and surgical factors to predict postoperative outcomes (Table 1). One of the most widely used is the Portsmouth Physiological and Operative Severity Score for the enUmeration of Mortality and morbidity (P-POSSUM), comprised of twelve physiological variables and six operative variables. The Estimation of Physiologic Ability and Surgical Stress (E-PASS) is another system calculated from six preoperative variables and three intraoperative variables [17]. In the United States, the National Surgical Quality Improvement Program (NSQIP) was developed to allow comparison of risk-adjusted surgical outcome data between hospitals. The risk prediction requires collection of 97 perioperative variables [21]. Finally, the Surgical Apgar Score (SAS) was derived to provide a simple postoperative assessment of patients at the end of a surgical procedure. The score is calculated from three intraoperative variables and predicts those patients at highest risk of postoperative complications and mortality [22]. All of these surgery-specific scoring systems can be applied to patients postoperatively irrespective of patient location. Indeed, none use ICU admission as a variable or as an outcome measure.

A major concern is that most of these scoring systems were created and evaluated for the purpose of audit, benchmarking, and assessment of quality of care. As such, they are valid for predicting outcomes in groups of patients, but they may not be useful or accurate for individual patients [18]. In addition, some of the scores, such as SOFA, MODS, and SAS, are much easier to implement in real time compared with others (such as NSQIP) to allow for an understanding of a patient's current status and to potentially assist in individual triage decisions.

\section{Postoperative complications}

Despite increasing recognition of the importance of postoperative complications as outcomes, previous research and scoring systems primarily focused on short-term (hospital or 30-day) mortality as the main postoperative outcome measure [18]. However, complications potentially result in greater morbidity for patients, increased length of hospital stay, and higher hospital costs. The focus in U.S. healthcare has recently shifted towards an increased emphasis on measuring complications because of a change in hospital reimbursements in 2008 by the Centers for Medicaid and Medicare Services (CMS). The CMS implemented a pay-for-performance initiative 
designed to improve the quality of health care delivered to patients. Hospitals no longer receive additional reimbursement when Medicare patients experience certain 'preventable' complications, some of which are clearly postoperative concerns, such as mediastinitis after cardiac surgery or an object retained inside the patient after surgery [23]. While increasing attention to these outcomes, it is unclear whether these economic incentives will result in a reduced incidence of postoperative complications.

Data also suggest a link between the development of postoperative complications and long-term outcomes. In recognition of the potential importance of complications, NSQIP provides prospective data collection on postoperative morbidity as well as mortality. Using the NSQIP dataset merged with a Veterans Benefits Administration database, a multicenter study of over 100,000 Veterans Affairs hospital patients undergoing eight different surgical procedures demonstrated that the presence of any complication within the first 30 postoperative days was an important predictor of 5-year survival, independent of preoperative risk [24]. Recognition and avoidance of postoperative complications may thus reduce shortterm morbidity and costs of care, and also impact longterm outcomes.

\section{"Failure to rescue"}

In two large studies of U.S. hospitals, Ghaferi et al. found that patients undergoing general and vascular surgical procedures had similar postoperative complication rates across hospitals but a very wide range of postoperative 30 -day mortality rates $[25,26]$. The rates of "failure to rescue" (proportion of deaths in patients who developed a postoperative complication out of the total number of patients who developed a postoperative complication) were much higher in high-mortality hospitals. The authors suggest that rather than differences in complication rates, it is differences in rates of "failure to rescue" that better explain hospital variation in post-surgical mortality; those hospitals with higher mortality may not properly recognize and manage postoperative complications when they occur $[25,26]$. This concept of "failure to rescue" shifts some of the focus from preventing the development of complications to improving the care provided to manage complications.

\section{Intensive care triage}

\section{Improved outcomes with intensive care}

Routine postoperative care in an ICU after high-risk surgical procedures may allow for greater recognition and correct management of postoperative complications, thereby reducing long-term morbidity and mortality. While there is an assumption that ICU admission may improve postoperative outcomes, no randomized trials have addressed this issue. Given the limitations and variability of ICU bed availability worldwide [27], many patients referred for ICU admission must ultimately be refused. The potential benefit of intensive care in reducing mortality for some groups of high-risk surgical patients is, therefore, suggested by multiple observational studies primarily examining patients refused admission to an ICU. In one prospective study that took place in seven countries, ICU admission of mixed medical and surgical patients was associated with a substantial reduction in 28- and 90-day mortality compared with patients refused admission. However, surgical patients were significantly less likely to be refused admission to an ICU than medical patients [28]. This discrepancy may be explained by the fact that many patients with severe systemic illness who undergo surgical procedures for acute problems are still deemed to have a reasonable chance of recovery. Moreover, curative surgery often represents an investment of resources in a patient that may carry over to priority admission to an ICU.

Until recently, few studies emphasized the potentially different nature and practice of triage decisions for postoperative patients compared with medical patients. One recent study of patients in two British hospitals that did focus solely on postoperative admission practices for high-risk surgical patients found that only one-third of these patients received intensive care. Furthermore, patients admitted to the ICU immediately after surgery had greatly improved survival compared with patients who were re-admitted or had delayed admission to the ICU postoperatively [29]. This study showed the potential drawbacks of ICU underuse, but it did not include details of the triage decision-making process for postoperative ICU admission. Objective, evidence-based criteria for ICU admission after surgery may facilitate resource allocation and potentially help identify patients who would benefit most from admission to the ICU, with the ultimate goal of improving postoperative outcomes.

\section{ICU admission guidelines}

In 1994, the European Society of Intensive Care Medicine (ESICM) issued guidelines stating that patients should be admitted to the ICU if they have an unstable condition or if they are at high risk of developing a severe complication [30]. The American College of Critical Care Medicine then published broad guidelines for ICU admission in 1999 [31]. A prioritization model was described to aid with decision-making, ranging from Priority 1 (patients who will derive the most benefit from admission) to Priority 4 (those who will not benefit at all). Priority 1 patients include hemodynamically unstable or ventilatordependent postoperative patients; Priority 2 patients are those who may need immediate intervention, such as patients with chronic comorbidities with an acute surgical problem [31]. The Italian intensivist group 
SIAARTI (Società Italiana di Anestesia Analgesia Rianimazione e Terapia Intensiva) published similar guidelines with specific recommendations for prioritization. A patient currently requiring intensive care should have priority for ICU admission over a patient who only needs intensive monitoring. This group also outlined a scale from Priority 1 (maximum benefit expected) to Priority 4 (little or no benefit expected) for determining which patients should take precedence for ICU admission [32]. Israeli consensus guidelines recommend ICU admission for all postoperative vascular or major general surgery patients with severe underlying systemic disease [33].

None of these sets of criteria, however, offers guidance as to how to triage patients of similar acuity. One method to allocate scarce ICU resources might be on a first-come, first-served basis, as suggested by the American Thoracic Society [34]. Current guidelines and studies also do not specifically target surgical populations. There are no universal criteria for postoperative admission of patients to the ICU. In a recent editorial, Goldhill and Down observed that postoperative critical care is highly inconsistent, with some groups of patients routinely admitted to the ICU postoperatively and others rarely admitted [35]. In some hospitals, patients are admitted postoperatively simply for monitoring, but it is unclear whether using the ICU for this purpose actually improves postoperative outcomes. While one early study found that a shortage of medical ICU beds led to restriction of admissions solely for monitoring purposes with no effect on mortality [36], no such study has been performed in surgical patients.

\section{Alternatives to ICU admission}

Mandatory ICU admission could potentially lead to overuse of intensive care for postsurgical patients, and a shift has occurred away from this practice. As an alternative to intensive care, the high-dependency unit (HDU) provides a location for patients with a potential but low risk of major complications who require more care than the ward but less than that available in the ICU [37]. Over a 14-year period from 1991 to 2004, postoperative care of major vascular surgery patients in one center changed from mandatory ICU admission for all patients to care for more than two-thirds of postoperative patients in an HDU and ward [38]. Another group retrospectively examined patients who had undergone radical cystectomy with urinary diversion followed by mandatory admission to the ICU postoperatively. They identified a stratification system for triage of these patients to the ICU, HDU, or ward depending on the risk of requiring active treatment such as invasive monitoring, vasoactive medications, or mechanical ventilation. However, there was no prospective evaluation of this postoperative risk stratification system [39]. Finally, an eight-year observational study compared ICU admissions for aortic abdominal surgery and surgery for lung cancer, two high-risk surgical procedures, before and after expansion of the hospital's post-anesthesia care unit (PACU). Admission rates to the ICU dropped significantly for both types of procedures, with no concomitant increase in postoperative morbidity or mortality [40]. These studies illustrate the possibility of caring for highrisk surgical patients in locations other than the ICU and highlight the potential for development of procedurespecific criteria for postoperative ICU admission.

\section{Post-anesthesia care units}

Individual hospital resources are extremely variable and not all centers have access to HDU facilities or proficient ward services. The PACU may often be used as an 'overflow' location for postoperative surgical patients who are not stable enough for ward care. Several issues may arise when a PACU is used to care for more severely ill patients. Physician coverage of these patients may be unclear, as anesthesia personnel, the primary surgical team, and/or an intensivist may all be involved in caring for such patients. In addition, PACU nurses must be fully competent and trained to care for ICU-level patients. Staffing patterns may need to be augmented, with increases in services from different provider types, such as respiratory therapists. Responsibility for recordkeeping, daily progress notes, ordering systems, and flowsheets must all be clarified [41]. While the PACU can serve as a safety net for ICU patients during times of limited bed availability, patients who would most benefit from postoperative ICU care would ideally gain admission to the ICU immediately after surgery, as outcomes may be improved with immediate rather than delayed ICU admission [29]. Therefore, for high-risk patients, bypassing the PACU for immediate transfer to intensive care may ensure the most efficient use of resources with the best outcomes.

\section{Improvement of postoperative outcomes}

A number of interventions have the potential to improve postoperative outcomes, especially in high-risk patients.

\section{Patient interventions}

Several studies show that hemodynamic optimization of high-risk surgical patients improves outcomes after surgery. The goal of hemodynamic optimization is to most efficiently match oxygen delivery to oxygen consumption. Tissue hypoxia due to surgical stress may not be adequately monitored by heart rate, blood pressure, or central venous pressure. Various invasive and noninvasive devices provide alternate means of assessing and optimizing oxygen delivery, including mixed or central venous oxygen saturation, cardiac index, pulse contour 
analysis, or other parameters indicating preload. Many of the studies using goal-directed hemodynamic therapy show decreased rates of postoperative complications, mortality, and hospital length-of-stay [42]. Individualized goal-directed therapy for high-risk surgical patients may improve outcome, but large, multicenter trials are necessary before widespread adoption can occur. British consensus guidelines, however, do recommend preoperative therapy with intravenous fluids and inotropes to attain predetermined cardiac output and oxygen delivery targets in high-risk surgical patients as a way to reduce postoperative mortality [43].

\section{Hospital interventions}

A simple way of improving surgical outcomes may include hospital-mandated use of a preoperative surgical safety checklist. A prospective study in eight countries looked at the rate of postoperative complications before and after implementing a checklist in all operating rooms. The checklist encompassed 19 items to ensure patient safety before induction of anesthesia, before surgical incision, and before exit from the operating room. All sites had reductions in major complication and mortality rates after implementation of the checklist [44].

Another method to reduce morbidity and mortality may be to improve ICU triage after surgery by creating and adhering to hospital guidelines that most efficiently use intensive care resources. These guidelines could include patient- and surgery-specific indications for admission to the ICU, HDU, or ward, and they could also delineate an ICU prioritization system for patients of similar acuity. 'Fast-track' surgery is a recent concept encompassing multimodal pre-, intra-, and postoperative interventions to improve outcomes after surgery. Each of these approaches must be specific to the surgical procedure and focus on enhancing postoperative recovery. Data are accumulating in a variety of surgical subspecialties that support the feasibility of 'fast-track' surgery [45]. Although practices vary, 'fast-track' surgery may mean more rapid progression through intensive care or admission to a specialized PACU.

Use of dedicated intensivists to staff ICUs may also help improve outcomes for high-risk surgical patients. In particular, intensivists may be the most appropriate practitioners to assist in ICU triage and bed allocation. Furthermore, dedicated intensivists may provide better care to ICU patients than non-specialized physicians. A survey of Maryland ICUs that provide postoperative care for patients undergoing abdominal aortic surgery showed that after adjusting for patient and hospital characteristics, the absence of daily rounds by an intensivist was associated with significantly increased morbidity and mortality after this high-risk surgery [46]. Similarly, rapid response teams with critical care-trained personnel may evaluate patients with complications on the ward to better identify those who might benefit from transfer to an ICU. Such teams may improve "failure-to-rescue" rates. Based on this concept, many hospitals have created rapid response teams [47], although their utility remains unproven in large multicenter studies.

\section{Health care system interventions}

On a health care system level, high volume hospitals and regionalization may also decrease the risk of postoperative morbidity and mortality. In an analysis of Medicare data, hospitals that performed a greater number of specific high-risk surgical procedures annually had lower hospital mortality rates [3]. A meta-analysis of the effects of surgical volume and specialization recently showed that high individual surgeon volume and surgeon specialization were independently associated with improved postoperative outcomes, but the relationship between high hospital volume overall and outcome remained unclear [48]. Regionalization to high-volume centers or to individual specially trained surgeons for certain high-risk procedures may be another way to improve outcomes after surgery, as is now the model for trauma care [49].

\section{Conclusion}

High-risk surgical patients continue to make up a substantial proportion of ICU admissions in most developed countries. Identification and optimization of these patients prior to surgical interventions remains difficult. Pre-, intra-, and postoperative variables all play a role in the development of considerable morbidity and mortality for high-risk patients. Appropriate decisions relating to the need for intensive care after surgery are key for highquality patient care, yet adequate systems for triage remain elusive. The potential exists for underuse of critical care resources, with inappropriate lack of admission to an ICU for high-risk patients, as well as overuse, with unnecessary admissions leading to increased length of stay and costs. With the wealth of data now available regarding the perioperative status of many of these patients, further research is needed to help facilitate optimal care for postsurgical patients.

\footnotetext{
Competing interests

The authors declare that they have no competing interests.

List of abbreviations used

ACCF: American College of Cardiology Foundation; AHA: American Heart Association; APACHE: acute physiology and chronic health evaluation; ASA: American Society of Anesthesiologists; CMS: Centers for Medicaid and Medicare Services; E-PASS: estimation of physiologic ability and surgical stress; ESICM: European Society of Intensive Care Medicine; HDU: high-dependency unit; ICU: intensive care unit; MODS: multiple organ dysfunction score; MPM: mortality probability model; NSQIP: national surgical quality improvement program; PACU: post-anesthesia care unit; P-POSSUM: portsmouth physiological and operative severity score for the enumeration of mortality and morbidity; RCRI: revised cardiac risk index; SAPS: simplified acute physiology score; SAS: surgical apgar score; SOFA: sequential organ failure assessment.
} 
Published: 22 March 2011

\section{References}

1. Nathanson BH, Higgins TL, Kramer AA, Copes WS, Stark M, Teres D: Subgroup mortality probability models: are they necessary for specialized intensive care units? Crit Care Med 2009, 37:2375-2386.

2. Pearse RM, Harrison DA, James P, et al:: Identification and characterisation of the high-risk surgical population in the United Kingdom. Crit Care 2006 10:R81.

3. Birkmeyer JD, Siewers AE, Finlayson EV, et al:. Hospital volume and surgical mortality in the United States. N Engl J Med 2002, 346:1128-1137.

4. Owens WD, Felts JA, Spitznagel EL Jr: ASA physical status classifications: a study of consistency of ratings. Anesthesiology 1978, 49:239-243.

5. Wolters U, Wolf T, Stutzer H, Schroder T: ASA classification and perioperative variables as predictors of postoperative outcome. Br J Anaesth 1996, 77:217-222

6. Charlson ME, Pompei P, Ales KL, MacKenzie CR: A new method of classifying prognostic comorbidity in longitudinal studies: development and validation. J Chronic Dis 1987, 40:373-383.

7. Lee TH, Marcantonio ER, Mangione CM, et al:: Derivation and prospective validation of a simple index for prediction of cardiac risk of major noncardiac surgery. Circulation 1999, 100:1043-1049.

8. McNicol L, Story DA, Leslie K, et al:: Postoperative complications and mortality in older patients having non-cardiac surgery at three Melbourne teaching hospitals. Med J Aust 2007, 186: 47-452.

9. Turrentine FE, Wang H, Simpson VB, Jones RS: Surgical risk factors, morbidity, and mortality in elderly patients. J Am Coll Surg 2006, 203:865-877.

10. Fleisher LA, Beckman JA, Brown KA, et al: 2009 ACCF/AHA focused update on perioperative beta blockade incorporated into the ACC/AHA 2007 guidelines on perioperative cardiovascular evaluation and care for noncardiac surgery: a report of the American college of cardiology foundation/American heart association task force on practice guidelines. Circulation 2009, 120:e169-e276.

11. Leung JM, Dzankic S: Relative importance of preoperative health status versus intraoperative factors in predicting postoperative adverse outcomes in geriatric surgical patients. J Am Geriatr Soc 2001, 49:1080-1085.

12. Aust JB, Henderson W, Khuri S, Page CP: The impact of operative complexity on patient risk factors. Ann Surg 2005, 241:1024-1027.

13. Reich DL, Bennett-Guerrero E, Bodian CA, Hossain S, Winfree W, Krol M: Intraoperative tachycardia and hypertension are independently associated with adverse outcome in noncardiac surgery of long duration. Anesth Analg 2002, 95:273-277.

14. Hartmann B, Junger A, Rohrig R, et al: Intra-operative tachycardia and perioperative outcome. Langenbecks Arch Surg 2003, 388:255-260.

15. Monk TG, Saini V, Weldon BC, Sigl JC: Anesthetic management and one-year mortality after noncardiac surgery. Anesth Analg 2005, 100:4-10.

16. Bijker JB, van Klei WA, Vergouwe Y, et al: Intraoperative hypotension and 1-year mortality after noncardiac surgery. Anesthesiology 2009, 111:1217-1226.

17. Chandra A, Mangam S, Marzouk D: A review of risk scoring systems utilised in patients undergoing gastrointestinal surgery. J Gastrointest Surg 2009, 13:1529-1538.

18. Higgins $T L:$ Quantifying risk and benchmarking performance in the adult intensive care unit. J Intensive Care Med 2007, 22:141-156.

19. Vincent JL, Moreno R, Takala J, et al:: The SOFA (Sepsis-related Organ Failure Assessment) score to describe organ dysfunction/failure. On behalf of the Working Group on Sepsis-Related Problems of the European Society of Intensive Care Medicine. Intensive Care Med 1996, 22:707-710.

20. Marshall JC, Cook DJ, Christou NV, Bernard GR, Sprung CL, Sibbald WJ: Multiple organ dysfunction score: a reliable descriptor of a complex clinical outcome. Crit Care Med 1995, 23:1638-1652.

21. Khuri SF, Henderson WG, Daley J, et al.: The patient safety in surgery study: background, study design, and patient populations. J Am Coll Surg 2007, 204:1089-1102.

22. Gawande AA, Kwaan MR, Regenbogen SE, Lipsitz SA, Zinner MJ: An Apgar score for surgery. J Am Coll Surg 2007, 204:201-208.

23. Morse BC, Boland BN, Blackhurst DW, Roettger RH: Analysis of Centers for Medicaid and Medicare Services 'never events' in elderly patients undergoing bowel operations. Am Surg 2010, 76:841-845.

24. Khuri SF, Henderson WG, DePalma RG, Mosca C, Healey NA, Kumbhani DJ: Determinants of long-term survival after major surgery and the adverse effect of postoperative complications. Ann Surg 2005, 242:326-341.
25. Ghaferi AA, Birkmeyer JD, Dimick JB: Complications, failure to rescue, and mortality with major inpatient surgery in medicare patients. Ann Surg 2009, 250:1029-1034.

26. Ghaferi AA, Birkmeyer JD, Dimick JB: Variation in hospital mortality associated with inpatient surgery. N Eng/J Med 2009, 361:1368-1375.

27. Wunsch $H$, Angus DC, Harrison DA, et al.: Variation in critical care services across North America and Western Europe. Crit Care Med 2008, 36:2787-2793.

28. lapichino G, Corbella D, Minelli C, et al: Reasons for refusal of admission to intensive care and impact on mortality. Intensive Care Med 2010, 36: 772-1779.

29. Jhanji S, Thomas B, Ely A, Watson D, Hinds CJ, Pearse RM: Mortality and utilisation of critical care resources amongst high-risk surgical patients in a large NHS trust. Anaesthesia 2008, 63:695-700.

30. European Society of Intensive Care Medicine: Guidelines for the utilisation of intensive care units. Intensive Care Med 1994, 20:163-164.

31. Task Force of the American College of Critical Care Medicine, Society of Critical Care Medicine: Guidelines for intensive care unit admission, discharge, and triage. Crit Care Med 1999, 27:633-638.

32. SIAARTI: SIAARTI guidelines for admission to and discharge from Intensive Care Units and for limitation of treatment in intensive care. Minerva Anestesiol 2003, 69:101-111.

33. Simchen E, Sprung CL, Galai N, et al: Survival of critically ill patients hospitalized in and out of intensive care. Crit Care Med 2007, 35:449-457.

34. American Thoracic Society: Fair allocation of intensive care unit resources. Am J Respir Crit Care Med 1997, 156:1282-1301.

35. Goldhill DR, Down JF: Are we operating as well as we can? Critical care to minimise postoperative mortality and morbidity. Anaesthesia 2008, 63:689-692.

36. Singer DE, Carr PL, Mulley AG, Thibault GE: Rationing intensive care-physician responses to a resource shortage. N Engl J Med 1983, 309:1155-1160.

37. Nasraway SA, Cohen IL, Dennis RC, et al:: Guidelines on admission and discharge for adult intermediate care units. American College of Critical Care Medicine of the Society of Critical Care Medicine. Crit Care Med 1998, 26:607-610

38. Teli M, Morris-Stiff G, Rees JR, Woodsford PV, Lewis MH: Vascular surgery, ICU and HDU: a 14-year observational study. Ann R Coll Surg Eng/ 2008, 90:291-296

39. Dahm P, Tuttle-Newhall JE, Nimjee SM, Byrne RR, Yowell CW, Price DT: Indications for admission to the surgical intensive care unit after radical cystectomy and urinary diversion. J Urol 2001, 166:189-193.

40. Schweizer A, Khatchatourian G, Hohn L, Spiliopoulos A, Romand J, Licker M: Opening of a new postanesthesia care unit: impact on critical care utilization and complications following major vascular and thoracic surgery. J Clin Anesth 2002, 14:486-493.

41. Lindsay $\mathrm{M}$ : Is the postanesthesia care unit becoming an intensive care unit? J Perianesth Nurs 1999, 14:73-77.

42. Kirov MY, Kuzkov WV, Molnar Z (2010) Perioperative haemodynamic therapy. Curr Opin Crit Care 16: 384-392

43. Soni N: British Consensus Guidelines on Intravenous Fluid Therapy for Adult Surgical Patients (GIFTASUP): Cassandra's view. Anaesthesia 2009, 64:235-238.

44. Haynes $A B$, Weiser $T G$, Berry WR, et al: A surgical safety checklist to reduce morbidity and mortality in a global population. N Engl J Med 2009, 360:491-499.

45. Kehlet H: Multimodal approach to postoperative recovery. Curr Opin Crit Care 2009, 15:355-358.

46. Pronovost PJ, Jenckes MW, Dorman T, et al:: Organizational characteristics of intensive care units related to outcomes of abdominal aortic surgery. JAMA 1999, 281:1310-1317.

47. Bellomo R, Goldsmith $D$, Uchino S, et al:: Prospective controlled trial of effect of medical emergency team on postoperative morbidity and mortality rates. Crit Care Med 2004, 32:916-921.

48. Chowdhury MM, Dagash $H$, Pierro A: A systematic review of the impact of volume of surgery and specialization on patient outcome. Br J Surg 2007. 94:145-161.

49. Kahn JM, Branas CC, Schwab CW, Asch DA: Regionalization of medical critical care: what can we learn from the trauma experience? Crit Care Med 2008, 36:3085-3088

doi:10.1186/cc9999

Cite this article as: Sobol JB, Wunsch H: Triage of high-risk surgical patients for intensive care. Critical Care 2011, 15:217. 